\title{
Phosphorus forms and distributions in soils under selected land use practices at the Federal University of Agriculture, Abeokuta, Ogun State, Nigeria
}

\section{Oladoye Abiodun Olusegun ${ }^{1 *}$, Busari Mutiu Abolanle', Abiodun Femi Olusola ${ }^{2}$, Ojekunle Oluseyi Opeyemi', Oyebamiji Noah Alabi ${ }^{3}$ and Olaife Blessing Adegboyega'}

The forms and distributions of phosphorus were determined for four different land use practices in Federal University of Agriculture, Abeokuta, with the aim of evaluating the effects of land use and soil depth on soil physical and chemical properties, forms and distributions of phosphorus. The study was a $4 \times 2$ factorial experiment laid out in a Randomized Complete Block Design. Factor 1 was four different land use practices (Gmelina arborea, Tectona grandis, Leucaena leucocephala plantations and secondary forest), and Factor 2 was soil samples collected from two soil depths $(0-30 \mathrm{~cm}$ and $30-60 \mathrm{~cm})$. The soil $\mathrm{pH}$, electrical conductivity, exchangeable bases ( $\mathrm{Na}, \mathrm{K}, \mathrm{Mg}$ and $\mathrm{Ca}$ ), particle size, organic carbon, total nitrogen, total phosphorus, inorganic phosphorus fractions (Al-P, Fe$\mathrm{P}$ and $\mathrm{Al}-\mathrm{P}$ ) and available phosphorus analyses were carried out using standard procedures. Data collected from the laboratory analysis were subjected to twoway Analysis of Variance while the means among the treatments were separated using Duncan Multiple Range Test. The results showed that the soils had low total and available P contents. The soil under Leucaena leucocephala plantation had the highest total phosphorus $\left(111.05 \mathrm{mg} \mathrm{kg}^{-1}\right)$, followed by Tectona grandis (97.63 $\left.\mathrm{mg} \mathrm{kg}^{-1}\right)$, Gmelina arborea $\left(96.41 \mathrm{mg} \mathrm{kg}^{-1}\right)$ and secondary forest $(83.63 \mathrm{mg}$ $\mathrm{kg}^{-1}$ ). Total P decreased with depths $(0-30 \mathrm{~cm}$ and $30-60 \mathrm{~cm})$. Inorganic P-fractions were greatly influenced by soil properties such as soil $\mathrm{pH}$, soil electrical conductivity, organic carbon, and soil particle size. The inorganic phosphorus fractions of the soils in all study area occurred generally in the following sequential order; Fe-P>Ca-P>Al-P. The highest inorganic phosphorus recorded

Federal University of Agriculture, Abeokuta, Nigeria

${ }^{2}$ Forestry Research Institute of Nigeria, Ibadan, Nigeria

${ }^{3}$ Federal University Dutsin-Ma, Katsina State, Nigeria

*Corresponding Author. Address: Federal University of Agriculture, Abeokuta, Nigeria; Email: oladoyeao@funaab.edu.ng 
in all in the study area was Fe-P, followed by Ca-P and Al-P. Al-P had the lowest content between the depths examined. Lower nutrient concentrations in soils under secondary forest within the depths were due to lower organic matter content, higher nutrient demand, nutrient immobilization by tree species and other flora present. $\mathrm{P}$ fractionation investigations are therefore recommended in soils under indigenous species plantation in order to provide adequate understanding of $P$ status in the soils and fertilizer recommendation so as to improve productivity.

Keywords: Land use, soil, phosphorus, inorganic fractions and distribution

\section{INTRODUCTION}

Phosphorus $(P)$ is the second most important plant nutrient element to nitrogen $(\mathrm{N})$ in terms of its essentiality in increasing the biomass production by plant (Junbao et al 2014). P exists in two main forms in the soil: organic and inorganic forms (Busman et al 2002). The inorganic phosphorus $(P)$ exists in compounds with Aluminum (Al), Iron (Fe) and Calcium ( $\mathrm{Ca}$ ) in the soil, and they exhibit variation in their solubility and availability to plants. The chemical fractionation of soil inorganic $P$ provides a method for identifying the predominant individual forms of inorganic $P$ in soils. The most commonly soluble P includes Aluminum P (Al-P), Iron P (Fe-P), Occluded $P$, and Calcium $P$ (Ca-P) (Chang and Jackson 1957). The fixation of phosphorus is said to be a common phenomenon in tropical soils (Igwe 2001) due to its high volume of Iron (Fe) and Aluminum (Al) hydrous oxides. The pH of a soil determines the type of phosphorus fixation that occurs; acidic soils tend to fix either Iron (Fe) or Aluminum (Al) phosphorus compound, while alkaline fixes Calcium (Ca) phosphorus compound (Havlin et al 2001). It is important to study Phosphorus (P) forms and distribution because it has no substitute in the growth of plant. Phosphorus cannot exist alone; it combines with other elements to form phosphate thereby limiting available phosphorus $(P)$ in the soil. Understanding the trend or status of $\mathrm{P}$ availability in different land use within Federal University of Agriculture, Abeokuta, Ogun State, Nigeria under the different land use will ensure proper management of the soil with respect to available phosphorus $(P)$. The variations in crops' ability to utilize different forms of phosphorus that occurs in soils and the fundamental variation in soil types are some of the major factors affecting the proper understanding of the behaviors of phosphorus in soil. Studies have shown that soils in the tropics like in Nigeria are generally deficient in phosphorus ( $P$ ) (Igwe 2001). The deficiency in phosphorus will limit yield, hence, the need to investigate phosphorus forms and distribution and help to devise effective means of soil phosphorus management. Various land use will contain different levels and forms of phosphorus as a result of environmental factors and activities that have been carried out on the land. The knowledge on the total phosphorus and its various functions in soil of the different land use in Federal University of Agriculture, Abeokuta, Ogun State, Nigeria and their distribution and availability to plants are essential in the management of soil phosphorus level, and these also foster the right fertilizer application. Many researches on phosphorus distribution and forms have been carried out in different parts of Nigeria considering both depth along the genetic horizons and surface soil. In the past, the study of soil P forms has been seen as a possible way to explain many processes and changes occurring in plant- 
soil interactions. In Abia State, phosphorus forms and distribution were analyzed along the depth of genetic horizons in two different locations. Therefore, the need to have a detailed study of the forms of $P$ and their distribution in the soils under different land use in Federal University of Agriculture, Abeokuta, seems warranted and will enable effective management of phosphorus content in the concerned areas.

\section{MATERIALS AND METHODS}

\section{The Study Area}

As described by (Sam-Wobo and Mafiana 2005), Ogun State lies approximately between Longitude $2^{\circ} 30^{\prime} \mathrm{E}$ and $4^{\circ} 30^{\prime} \mathrm{E}$ and between Latitudes $6^{\circ} 30^{\prime} \mathrm{N}$ and $80^{\circ} \mathrm{N}$. It is located in the rainforest ecological zone of Nigeria. Four land uses located within Federal University of Agriculture, Abeokuta, Nigeria were used for the study. Federal University of Agriculture, Abeokuta lies approximately between Longitude 7.230938 E and Latitude 3.438232N within Abeokuta, Ogun State.

\section{Experimental Design}

The study was a $4 \times 2$ factorial experiment laid out in a Randomized Complete Block Design (RCBD) with factor 1 as the four different land uses (Leucaena leucocephala, Gmelina arborea, Tectona grandis plantations and a secondary forest) selected within the University and factor 2 was soil samples collected from two soil depths at each sample site which comprises of soils from $(0-30 \mathrm{~cm})$ and $(30-60 \mathrm{~cm})$ depths. Samples collected from both depths in the four land uses were replicated three times to give a total of 24 experimental units.

\section{Soil Preparation and Analysis}

The collected soil samples were stored immediately in polyethylene plastic bags before transporting to the laboratory. All soil samples were air-dried for 3 days at room temperature to remove moisture and then sieved through a $2 \mathrm{~mm}$ diameter sieve. The $\mathrm{pH}$ of the soil samples was determined in water by means of Bechman's $\mathrm{pH}$ meter using a soil water ratio of 1:2.5 (Thomas 1996). The particle size distributions of the soils were determined by the hydrometer method (Bouyoucos 1951). Organic carbon was determined by the procedure of Walkley and Black (1934) using the dichromate wet oxidation method (Nelson and Sommers 1996). The soils Exchangeable bases were extracted with $1 \mathrm{~N}$ Ammonium acetate (NH4OAc) at $\mathrm{pH7.0}$. $\mathrm{Ca}$ and $\mathrm{Mg}$ in the extracts were determined by titration (Jackson 1958) while $\mathrm{Na}$ and $\mathrm{K}$ were determined with flame photometer. The characterization of the different forms of inorganic phosphorus in soils was carried out by sequential extraction with acid and alkaline reagents as initiated by Dean (1938) which was later modified by Chang and Jackson (1957). The procedures used are modifications and improvements of the sequence, based on the extraction using acid and alkaline reagents, originally suggested by Chang and Jackson (Chang and Jackson 1957, Tiyapongpattana et al 2004) while the Phosphorus concentration in the various extracts was determined using the phosphomolybdate method (Murphy and Riley 1962). 


\section{Data Analysis}

The data were analysed using Statistical Analysis System (SAS) procedures and software (SAS 2003). The two-way analysis of variance (ANOVA) tables was constructed using the Generalized Linear Model (GLM) procedure. The significant means were separated by the Duncan's Multiple Range Test (Duncan 1955) at $p<0.05$.

\section{RESULTS}

The mean values of the sand particles of the soils at $0-30 \mathrm{~cm}$ and $30-60 \mathrm{~cm}$ depths were $87500 \pm 0.79$ and $862.50 \pm 0.72 \mathrm{~g} \mathrm{~kg}^{-1}$ which were not significantly different $(P=.05)$ from each other. Likewise, there was no significant difference $(P=.05)$ in the mean values of their clay $\left(\mathrm{g} \mathrm{kg}^{-1}\right)$ particles at both depths respectively with $72.50 \pm 0.18 \mathrm{~g} \mathrm{~kg}^{-1}$ recorded in both $0-30 \mathrm{~cm}$ and $30-60 \mathrm{~cm}$. The silt particles in the soils at $0-30 \mathrm{~cm}$ and $30-60 \mathrm{~cm}$ depth which were $5.2 .50 \pm 0.74 \mathrm{~g} \mathrm{~kg}^{-1}$ and $65.00 \pm 0.64 \mathrm{~g}$ $\mathrm{kg}^{-1}$ were also not significantly different $(P=.05)$ from each other (Table 1$)$. There was a significant difference $(P=.05)$ in the mean values of $\mathrm{pH}$ recorded at both soil depths. The highest $\mathrm{pH}$ value was recorded at $0-30 \mathrm{~cm}(6.89 \pm 0.06)$ and the least at $30-60 \mathrm{~cm}$ depth $(6.55 \pm 0.15)$ which can both be classified as slightly acidic. The electrical conductivity decreased with depths as soil at $0-30 \mathrm{~cm}$ recorded $0.19 \pm 0.01 \mathrm{ds} \mathrm{m}^{-1}$ which was significantly higher than $0.09 \pm 0.01 \mathrm{ds} \mathrm{m}^{-1}$ recorded at $30-60 \mathrm{~cm}$ soil depth (Table 1). Organic carbon also decreased down the depths with soil at $0-30 \mathrm{~cm}$ having the highest organic carbon $\left(17.00 \pm 0.13 \mathrm{~g} \mathrm{~kg}^{-1}\right)$ compared to $7.80 \pm 0.14 \mathrm{~g} \mathrm{~kg}^{-1}$ available at $30-60 \mathrm{~cm}$ which was significantly lower at $(P=.05)$. The total phosphorus down the depths of the studied soils was $99.51 \pm 3.21 \mathrm{mg} \mathrm{kg}^{-1}$ at 0 $30 \mathrm{~cm}$ and $94.85 \pm 4.15 \mathrm{mg} \mathrm{kg}^{-1}$ at $30-60 \mathrm{~cm}$ depths. The total phosphorus (T.P) contents of normal soils vary from 100 to $2000 \mathrm{mg} \mathrm{kg}^{-1}$ (Table 1). Calcium bound phosphate (Ca-P) form of phosphorus was $27.02 \pm 1.62 \mathrm{mg} \mathrm{kg}^{-1}$ and $27.88 \pm 1.74 \mathrm{mg}$ $\mathrm{kg}^{-1} 0-30 \mathrm{~cm}$ and $30-60 \mathrm{~cm}$ soil depths respectively, and there was no significant difference between the two depths while the highest mean of $\mathrm{Ca}-\mathrm{P}$ was recorded in soils at $30-60 \mathrm{~cm}$ depth. Iron bound phosphate result showed a significant difference between both depths while $44.07 \pm 1.96 \mathrm{mg} \mathrm{kg}^{-1}$ and $37.69 \pm 2.06 \mathrm{mg} \mathrm{kg}^{-1}$ were recorded at $0-30 \mathrm{~cm}$ and $30-60 \mathrm{~cm}$ soil depths respectively. Aluminum bound phosphate in the two depths were $18.38 \pm 0.49 \mathrm{mg} \mathrm{kg}^{-1}$ and $22.10 \pm 1.99 \mathrm{mg} \mathrm{kg}^{-1}$ respectively. The result shows there was no significant difference between the two depths, and the highest result recorded in the soil was at $30-60 \mathrm{~cm}$ depth. Available phosphorus recorded in both depths was $6.47 \pm 0.48 \mathrm{mg} \mathrm{kg}^{-1}$ and $6.77 \pm 1.06 \mathrm{mg} \mathrm{kg}^{-1}$ in $0-30 \mathrm{~cm}$ and $30-60 \mathrm{~cm}$ soil depths respectively. Also, there was no significance difference in the available phosphorus between the depths (Table 1).

In relation to land use practices, the soil $\mathrm{pH}$ result showed a significant difference between soils under Gmelina arborea plantation and secondary forest, both with a $\mathrm{pH}$ value of $6.9 \pm 0.08$ and $6.13 \pm 0.19$ respectively and also those of Tectona grandis, Leucaena leucocephala and secondary forest. The $\mathrm{pH}$ values recorded for Tectona grandis and Leucena leucocephana were $6.95 \pm 0.05$ and $6.89 \pm 0.04$, respectively (Table 2 ). The highest $\mathrm{pH}$ was recorded in soils under Tectona grandis with $6.95 \pm 0.05$ and that value was significantly higher than $6.13 \pm 0.19$ recorded under secondary forest (Table 1 ). A significantly higher 
electrical conductivity was recorded in soil under Leucaena leucocephala plantation $0.17 \pm 0.03 \mathrm{ds} \mathrm{m}^{-1}$ compared to all other soils considered in the study. The organic carbon percentages in the four locations were $12.30 \pm 0.28 \mathrm{~g} \mathrm{~kg}^{-1}, 15.70 \pm 0.01 \mathrm{~g} \mathrm{~kg}^{-1}$ $14.00 \pm 0.38 \mathrm{~g} \mathrm{~kg}^{-1}$ and $7.60 \pm 0.17 \mathrm{~g} \mathrm{~kg}^{-1}$ with that of Tectona grandis plantation being significantly higher than all others. The constant distribution of organic carbon in the soil under Tectona grandis plantation can be due to the equal deposition of organic matter in the soil. The soil organic matter accumulation has been shown to be proportional to the extent of canopy closure. The occurrence of higher soil organic carbon content under Tectona grandis plantation can be due to the litter fall addition from trees and shrubs to the surface soil. Similar trend was also observed from the result of total nitrogen obtained under Tectona grandis plantation (Tables 2 and 3 ). Leucaena plantation had the significantly higher total phosphorus $(111.45 \pm 3.32 \mathrm{mg}$ $\left.\mathrm{kg}^{-1}\right)$ at depth of $0-30 \mathrm{~cm}$ and the lowest in secondary forest $\left(79.85 \pm 10.77 \mathrm{mg} \mathrm{kg}^{-1}\right)$ at the depth of $30-60 \mathrm{~cm}$, which could be due to erosion, crop removal and precipitation of phosphorus from soil solution which decreases phosphorus availability, as Fe-P was found to be dominant in all the depth of Gmelina, Tectona, Leucaena plantations and secondary forest in which the highest Fe-P was found in Gmelina plantation $\left(48.1 \pm 2.31 \mathrm{mg} \mathrm{kg}^{-1}\right)$ at $0-30 \mathrm{~cm}$ and $47.08 \pm 2.68 \mathrm{mg} \mathrm{kg}^{-1}$ under Leucaena plantation at $0-30 \mathrm{~cm}$ depth. Fe-P and Al-P fraction of phosphorus were closely similar both having $28.19 \pm 5.71 \mathrm{mg} \mathrm{kg}^{-1}$ and $28.05 \pm 7.54 \mathrm{mg} \mathrm{kg}^{-1}$ respectively at $30-60 \mathrm{~cm}$ depth in secondary forest, while it varied in others (Table 3 ).

Table 1. Effect of depth on the soil physiochemical properties and forms of phosphorus

\begin{tabular}{|c|c|c|}
\hline \multirow{2}{*}{ Soil properties } & \multicolumn{2}{|c|}{ Soil depth (cm) } \\
\hline & $0-30$ & $30-60$ \\
\hline Sand\% & $87.5 \pm 0.79^{a}$ & $86.25 \pm 0.72^{a}$ \\
\hline Clay\% & $7.25 \pm 0.18{ }^{a}$ & $7.25 \pm 0.18^{a}$ \\
\hline Silt $\%$ & $5.25 \pm 0.74{ }^{a}$ & $6.50 \pm 0.64$ a \\
\hline $\mathrm{pH}\left(\mathrm{H}_{2} \mathrm{O}\right) 1: 2$ & $6.89 \pm 0.06$ a & $6.55 \pm 0.15 \mathrm{~b}$ \\
\hline $\mathrm{EC}\left(\mathrm{ds} \mathrm{m}^{-1}\right)$ & $0.19 \pm 0.01 \quad \mathrm{a}$ & $0.09 \pm 0.01 \mathrm{~b}$ \\
\hline $0 . C \%$ & $1.70 \pm 0.13$ a & $0.78 \pm 0.14 b$ \\
\hline T.N\% & $0.15 \pm 0.01 \quad a$ & $0.07 \pm 0.01$ a \\
\hline $\mathrm{Na}\left(\mathrm{cmol} \mathrm{kg}^{-1}\right)$ & $0.45 \pm 0.02 a^{a}$ & $0.49 \pm 0.03$ a \\
\hline $\mathrm{Ca}\left(\mathrm{cmol} \mathrm{kg}^{-1}\right)$ & $3.97 \pm 0.76{ }^{a}$ & $2.59 \pm 0.28 \mathrm{~b}$ \\
\hline $\mathrm{Mg}\left(\mathrm{cmol} \mathrm{kg}^{-1}\right)$ & $258 \pm 0.46$ a & $2.67 \pm 0.24$ a \\
\hline $\mathrm{K}\left(\mathrm{cmol} \mathrm{kg}^{-1}\right)$ & $0.05 \pm 0.01 \quad a$ & $0.04 \pm 0.01 \quad b$ \\
\hline Total $-\mathrm{P}\left(\mathrm{mg} \mathrm{kg}^{-1}\right)$ & $99.51 \pm 3.21$ a & $94.85 \pm 4.15$ a \\
\hline $\mathrm{Ca}-\mathrm{P}\left(\mathrm{mg} \mathrm{kg}^{-1}\right)$ & $27.02+1.62$ a & $27.88 \pm 1.74$ a \\
\hline $\mathrm{Fe}-\mathrm{P}\left(\mathrm{mg} \mathrm{kg}^{-1}\right)$ & $44.07 \pm 1.96$ a & $37.69 \pm 2.06 \mathrm{~b}$ \\
\hline Al-P $\left(\mathrm{mg} \mathrm{kg}^{-1}\right)$ & $18.38 \pm 0.49 a$ & $22.10 \pm 1.99$ a \\
\hline Available $-P\left(\mathrm{mg} \mathrm{kg}^{-1}\right)$ & $6.47 \pm 0.48$ & $6.77 \pm 1.06$ a \\
\hline
\end{tabular}

Values of the same alphabets horizontally are not significantly different at $(p \geq 0.05)$ 
Table 2. Effect of land use practices on the soil physiochemical properties and phosphorus forms of the study area

\begin{tabular}{|c|c|c|c|c|}
\hline Soil Properties & Gmelina plantation & Teak plantation & $\begin{array}{l}\text { Leucena } \\
\text { plantation }\end{array}$ & Secondary forest \\
\hline Sand\% & $86.50 \pm 0.73 \mathrm{bc}$ & $87.00 \pm 1.45 b$ & $84.50 \pm 0.00 \mathrm{c}$ & $89.50 \pm 0.37 \quad$ a \\
\hline Clay\% & $7.50 \pm 0.34$ a & $7.50 \pm 0.34$ a & $7.00 \pm 0.00 \mathrm{a}$ & $7.00 \pm 0.00$ \\
\hline Silt\% & $6.00 \pm 0.62 \mathrm{~b}$ & $5.50 \pm 1.21 \quad b$ & $8.50 \pm 0.00 \quad \mathrm{a}$ & $3.50 \pm 0.37{ }^{c}$ \\
\hline $\mathrm{pH}\left(\mathrm{H}_{2} \mathrm{O}\right) 1: 2$ & $6.90 \pm 0.08$ ab & $6.95 \pm 0.05 a$ & $6.89 \pm 0.04 \quad b$ & $6.13 \pm 0.19 c$ \\
\hline $\mathrm{ECdsm}^{-1}$ & $0.12+0.02 c$ & $0.16 \pm 0.02$ & $0.17 \pm 0.03 \quad$ a & $0.10 \pm 0.02 d$ \\
\hline $0 . C \%$ & $1.23 \pm 0.28 c$ & $1.57 \pm 0.01 \quad \mathrm{a}$ & $1.4 \pm 0.38 \mathrm{~b}$ & $0.76 \pm 0.17 d$ \\
\hline T.N\% & $0.11 \pm 0.02$ & $0.14 \pm 0.00$ a & $0.12+0.03 b$ & $0.07 \pm 0.01 d$ \\
\hline $\mathrm{Na}\left(\mathrm{cmol} \mathrm{kg}^{-1}\right)$ & $0.44 \pm 0.03 a b$ & $0.43 \pm 0.02 \quad b$ & $0.54 \pm 0.05{ }^{a}$ & $0.48 \pm 0.03$ ab \\
\hline $\mathrm{Ca}\left(\mathrm{cmol} \mathrm{kg}^{-1}\right)$ & $3.57 \pm 0.44$ b & $3.61 \pm 0.20 b$ & $5.04 \pm 1.07$ a & $0.9 \pm 0.30 \mathrm{c}$ \\
\hline $\mathrm{Mg}\left(\mathrm{cmol} \mathrm{kg}^{-1}\right)$ & $262+0.20 \mathrm{c}$ & $289 \pm 0.15 b$ & $4.05 \pm 0.20$ a & $0.93 \pm 0.33 d$ \\
\hline $\mathrm{K}\left(\mathrm{cmol} \mathrm{kg}^{-1}\right)$ & $0.05 \pm 0.01 \quad a$ & $0.04 \pm 0.01 \quad \mathrm{a}$ & $0.05 \pm 0.00 \mathrm{a}$ & $0.05 \pm 0.01 \quad \mathrm{a}$ \\
\hline Total $-P\left(\mathrm{mg} \mathrm{kg}^{-1}\right)$ & $96.41 \pm 4.15$ b & $97.63 \pm 1.51 \quad b$ & $111.05 \pm 1.74$ a & $83.63 \pm 5.24 \quad c$ \\
\hline $\mathrm{Ca}-\mathrm{P}\left(\mathrm{mgkg}^{-1}\right)$ & $29.76 \pm 2.10 \quad a$ & $25.55 \pm 2.14$ a & $29.98 \pm 1.28 \quad$ a & $24.5 \pm 3.11$ a \\
\hline $\mathrm{Fe}-\mathrm{P}\left(\mathrm{mgkg}^{-1}\right)$ & $44.43 \pm 1.94$ a & $44.03 \pm 1.51 \quad$ a & $44.01 \pm 1.83 \quad$ a & $31.07 \pm 295$ b \\
\hline Al-P(mgkg $\left.{ }^{-1}\right)$ & $17.67 \pm 0.55$ a & $19.39 \pm 0.98 \quad$ a & $21.14 \pm 0.41 \quad$ a & $2277 \pm 4.13$ a \\
\hline Available $-\mathrm{P}\left(\mathrm{mg} \mathrm{kg}^{-1}\right)$ & $5.24 \pm 0.49$ b & $6.00 \pm 0.94$ & $9.12+1.54$ a & $6.10 \pm 0.83 b$ \\
\hline
\end{tabular}

Values of the same alphabets horizontally are not significantly different at $(p \geq 0.05)$

The results of correlation studies on the soils showed a positive significant correlation of $\mathrm{pH}$ with Iron bound phosphate (Fe-P) $(r=0.79)$, calcium bound phosphate (Ca-P) $(r=0.28)$, total phosphorus $(r=0.62)$ and available phosphorus $(r=0.09)$. Also, $\mathrm{pH}$ showed positive correlation with electrical conductivity $(r=0.56)$, organic carbon $(r=0.57)$ and total nitrogen $(r=0.58)$. Likewise, result showed $p H$ also had a significant positive correlation with magnesium ( $r=0.48)$, calcium $(r=0.47)$, potassium $(r=0.42)$. $\mathrm{pH}$ also showed a positive significant correlation with silt $(r=0.37)$ and clay $(r=0.26)$.

Electrical conductivity showed a positive correlation with percentage organic carbon $(r=0.84)$, total nitrogen $(r=0.84)$, total phosphorus $(r=0.47)$, iron bound phosphate $(r=0.63)$, calcium $(r=0.72)$, and magnesium $(r=0.44)$. Result also showed that percentage organic carbon has a significant positive correlation with total nitrogen $(r=0.99)$, total phosphorus $(r=0.45)$, iron bound phosphate (Fe-P) $(r=0.64)$. Also, percentage organic carbon showed a positive correlation with calcium $(r=0.75)$ and magnesium $(r=0.49)$.

Sand also showed negative correlation with exchangeable calcium ( $r=-0.42)$, and magnesium $(r=-0.63)$. Silt also showed a significant positive correlation with total phosphorus $(r=0.55)$, calcium bound phosphate $(C a-P)(r=0.43)$. Also, silt had a positive correlation with calcium $(r=0.42)$ and magnesium $(r=0.65)$. Total phosphorus had a significant positive correlation with Calcium bound phosphate $(\mathrm{Ca}-\mathrm{P})(\mathrm{r}=0.59)$, Iron bound phosphate (Fe-P) $(r=0.81)$, exchangeable base Calcium $(r=0.60)$, and magnesium ( $r=0.67)$. However, there was a negative significant correlation between total phosphorus and aluminum bound phosphate $(r=-0.47)$. 
Table 3. Interactive effect of land use practices and depth on the soil physiochemical properties and phosphorus forms of the study area

\begin{tabular}{|c|c|c|c|c|c|c|c|c|}
\hline Location/depth & $\begin{array}{c}\text { Gmelina } \\
\text { plantation } \\
0-30 \mathrm{~cm}\end{array}$ & $\begin{array}{c}\text { Gmelina } \\
\text { plantation } \\
30-60 \mathrm{~cm}\end{array}$ & $\begin{array}{c}\text { Teak } \\
\text { plantation } \\
0-30 \mathrm{~cm}\end{array}$ & $\begin{array}{c}\text { Teak } \\
\text { plantation } \\
30-60 \mathrm{~cm}\end{array}$ & $\begin{array}{l}\text { Leucena } \\
\text { plantation } \\
0-30 \mathrm{~cm}\end{array}$ & $\begin{array}{l}\text { Leucena } \\
\text { plantation } \\
30-60 \mathrm{~cm}\end{array}$ & $\begin{array}{c}\text { Secondary } \\
\text { Forest } \\
0-30 \mathrm{~cm}\end{array}$ & $\begin{array}{l}\text { Secondary } \\
\text { Forest } \\
30-60 \mathrm{~cm}\end{array}$ \\
\hline Sand\% & $86.50 \pm 1.15$ ab & $86.50 \pm 1.15 \mathrm{ab}$ & $89.50 \pm 1.73$ a & $84.50 \pm 1.15^{b}$ & $84.50 \pm 0.00^{b}$ & $84.50 \pm 0.00^{b}$ & $89.50 \pm 0.58$ a & $89.50 \pm 0.588^{a}$ \\
\hline Clay\% & $8.00 \pm 0.588^{a}$ & $7.00 \pm 0.00^{b}$ & $7.00 \pm 0.00^{b}$ & $8.00 \pm 0.588^{a}$ & $7.00 \pm 0.00^{b}$ & $7.00 \pm 0.00^{b}$ & $7.00 \pm 0.00 \mathrm{~b}$ & $7.00 \pm 0.00 \mathrm{~b}$ \\
\hline Silt\% & $5.50 \pm 0.58 \mathrm{bc}$ & $6.50 \pm 1.15 \mathrm{ab}$ & $3.50 \pm 1.73^{c}$ & $7.50 \pm 0.58 \mathrm{ab}$ & $8.50 \pm 0.00^{a}$ & $8.50 \pm 0.00^{a}$ & $3.50 \pm 0.58 c$ & $3.50 \pm 0.58 \mathrm{c}$ \\
\hline $\mathrm{pH}\left(\mathrm{H}_{2} \mathrm{O}\right) 1: 2$ & $7.08 \pm 0.01^{a}$ & $6.73 \pm 0.02 \mathrm{~d}$ & $7.05 \pm 0.01$ a & $6.85 \pm 0.03^{c}$ & $6.84 \pm 0.02^{c}$ & $6.94 \pm 0.06^{b}$ & $6.57 \pm 0.01$ e & $5.68 \pm 0.01^{f}$ \\
\hline E. $C\left(\mathrm{ds} \mathrm{m}^{-1}\right)$ & $0.15 \pm 0.00^{c}$ & $0.09 \pm 0.00 \mathrm{~g}$ & $0.21 \pm 0.00 \mathrm{~b}$ & $0.11 \pm 0.00 e^{e}$ & $0.25 \pm 0.00^{a}$ & $0.10 \pm 0.00^{f}$ & $0.14 \pm 0.00 \mathrm{~d}$ & $0.06 \pm 0.00 \mathrm{~h}$ \\
\hline $0 . C \%$ & $1.86 \pm 0.06^{b}$ & $0.60 \pm 0.02 e^{e}$ & $1.57 \pm 0.02^{c}$ & $1.57 \pm 0.02^{c}$ & $2.24 \pm 0.06^{a}$ & $0.56 \pm 0.08$ ef & $1.12 \pm 0.10^{d}$ & $0.40 \pm 0.01^{f}$ \\
\hline T.N $\%$ & $0.16 \pm 0.01 \mathrm{~b}$ & $0.06 \pm 0.00 \mathrm{e}$ & $0.14 \pm 0.00^{c}$ & $0.14 \pm 0.00^{c}$ & $0.19 \pm 0.01^{a}$ & $0.05 \pm 0.01$ ef & $0.10 \pm 0.01 \mathrm{~d}$ & $0.04 \pm 0.00^{f}$ \\
\hline $\mathrm{Na}\left(\mathrm{cmol} \mathrm{kg}{ }^{-1}\right)$ & $0.50 \pm 0.03 \mathrm{ab}$ & $0.39 \pm 0.02 \mathrm{~b}$ & $0.38 \pm 0.01 \mathrm{~b}$ & $0.47 \pm 0.01 \mathrm{ab}$ & $0.47 \pm 0.02 \mathrm{ab}$ & $0.60 \pm 0.09$ a & $0.47 \pm 0.06 \mathrm{ab}$ & $0.49 \pm 0.01 \mathrm{ab}$ \\
\hline $\mathrm{Ca}\left(\mathrm{cmol} \mathrm{kg}{ }^{-1}\right)$ & $4.49 \pm 0.00 \mathrm{~b}$ & $2.65 \pm 0.31 \mathrm{~d}$ & $3.84 \pm 0.34 \mathrm{bc}$ & $3.39 \pm 0.12 \mathrm{~cd}$ & $7.29 \pm 0.00^{a}$ & $2.79 \pm 0.81 \mathrm{~cd}$ & $0.26 \pm 0.01^{f}$ & $1.55 \pm 0.14 \mathrm{e}^{\mathrm{e}}$ \\
\hline $\mathrm{Mg}\left(\mathrm{cmol} \mathrm{kg}^{-1}\right)$ & $3.04 \pm 0.02^{c}$ & $2.19 \pm 0.16^{e}$ & $2.58 \pm 0.09 \mathrm{~d}$ & $3.19 \pm 0.13^{c}$ & $4.48 \pm 0.08{ }^{a}$ & $3.62 \pm 0.13 b$ & $0.21 \pm 0.11 \mathrm{~g}$ & $1.66 \pm 0.10^{f}$ \\
\hline $\mathrm{K}(\mathrm{cmol} \mathrm{kg}$ & $0.06 \pm 0.01 \mathrm{ab}$ & $0.03 \pm 0.01 \mathrm{~cd}$ & $0.04 \pm 0.01$ bed & $0.05 \pm 0.01 \mathrm{abcd}$ & $0.05 \pm 0.01 \mathrm{abcd}$ & $0.06 \pm 0.06 \mathrm{abc}$ & $0.07 \pm 0.01$ a & $0.02 \pm 0.00^{d}$ \\
\hline Total $-\mathrm{P}\left(\mathrm{mg} \mathrm{kg}^{-1}\right)$ & $101.55 \pm 7.71 \mathrm{ab}$ & $91.3 \pm 0.81$ bc & $97.65 \pm 0.78 \mathrm{ab}$ & $97.6 \pm 3.29 \mathrm{ab}$ & $111.45 \pm 3.32$ a & $110.65 \pm 1.99$ a & $87.4 \pm 2.65$ bc & $79.85 \pm 10.77^{c}$ \\
\hline Ca-P $\left(\mathrm{mg} \mathrm{kg}^{-1}\right)$ & $32.59 \pm 1.43$ a & $26.93 \pm 3.48 \mathrm{ab}$ & $21.25 \pm 1.88 \mathrm{~b}$ & $29.86 \pm 0.95 a b$ & $29.6 \pm 2.66 \mathrm{ab}$ & $30.34 \pm 1.01 \mathrm{ab}$ & $24.63 \pm 2.64 \mathrm{ab}$ & $24.38 \pm 6.42 a b$ \\
\hline $\mathrm{Fe}-\mathrm{P}\left(\mathrm{mg} \mathrm{kg}{ }^{-1}\right)$ & $48.1 \pm 2.31^{a}$ & $40.76 \pm 0.23 \mathrm{ab}$ & $47.16 \pm 1.18$ a & $40.91 \pm 0.53$ ab & $47.08 \pm 2.68 \mathrm{a}^{\mathrm{a}}$ & $40.94 \pm 0.55 \mathrm{ab}$ & $33.95 \pm 1.58$ bc & $28.19 \pm 5.71^{\mathrm{c}}$ \\
\hline Al-P $\left(\mathrm{mg} \mathrm{kg}^{-1}\right)$ & $17.68 \pm 0.07 \mathrm{~b}$ & $17.66 \pm 1.24 \mathrm{~b}$ & $17.40 \pm 0.41 \mathrm{~b}$ & $21.38 \pm 0.82 \mathrm{ab}$ & $20.94 \pm 0.44$ ab & $21.33 \pm 0.78$ ab & $17.48 \pm 0.70 \mathrm{~b}$ & $28.05 \pm 7.54$ a \\
\hline Available $-\mathrm{Pmg} \mathrm{kg}^{-1}$ & $4.31 \pm 0.14 \mathrm{~b}$ & $6.18 \pm 0.55 \mathrm{~b}$ & $8.05 \pm 0.36 \mathrm{ab}$ & $3.96 \pm 0.26^{b}$ & $7.19 \pm 0.57$ ab & $11.05 \pm 2.80^{\mathrm{a}}$ & $6.32 \pm 0.84 \mathrm{~b}$ & $5.89 \pm 1.64 \mathrm{~b}$ \\
\hline
\end{tabular}


Table 4. Correlation coefficient between soil physicochemical properties and phosphorus fractions

\begin{tabular}{|c|c|c|c|c|c|c|c|c|c|c|c|c|c|c|c|c|}
\hline & $\begin{array}{c}\mathrm{pH} \\
\left(\mathrm{H}_{2} 01: 2\right)\end{array}$ & $\begin{array}{l}\text { E.C } \\
\text { (ds } \\
m^{-1} \text { ) } \\
\end{array}$ & $0 . C \%$ & $\begin{array}{c}\text { Sand } \\
\%\end{array}$ & $\begin{array}{c}\text { Clay } \\
\%\end{array}$ & $\begin{array}{l}\text { Silt } \\
\%\end{array}$ & $\begin{array}{c}\text { T.N } \\
\%\end{array}$ & $\begin{array}{l}\text { Total - } \\
\text { P mg } \\
\mathrm{kg}^{-1} \\
\end{array}$ & $\begin{array}{c}\mathrm{Ca}-\mathrm{P} \\
\mathrm{mg} \\
\mathrm{kg}^{-1} \\
\end{array}$ & $\begin{array}{l}\text { Fe-P } \\
\mathrm{mg} \\
\mathrm{kg}^{-1} \\
\end{array}$ & $\begin{array}{l}\mathrm{Al}-\mathrm{P} \\
\mathrm{mg} \\
\mathrm{kg}^{-1} \\
\end{array}$ & $\begin{array}{c}\text { Available }-P \\
\text { mg } \\
\mathrm{kg}^{-1}\end{array}$ & $\begin{array}{c}\mathrm{Na} \\
\mathrm{cmol} \\
\mathrm{kg}^{-1} \\
\end{array}$ & $\begin{array}{c}\mathrm{Ca} \\
\mathrm{cmol} \\
\mathrm{kg}^{-1} \\
\end{array}$ & $\begin{array}{c}\mathrm{Mg} \\
\mathrm{cmoll} \\
\mathrm{kg}^{-1} \\
\end{array}$ & $\begin{array}{c}\mathrm{K} \\
\mathrm{cmol} \\
\mathrm{kg}^{-1} \\
\end{array}$ \\
\hline $\mathrm{pH}\left(\mathrm{H}_{2} \mathrm{O}\right) 1: 2$ & 1 & & & & & & & & & & & & & & & \\
\hline E.C $\left(\mathrm{ds} \mathrm{m}^{-1}\right)$ & $0.56^{\text {** }}$ & 1 & & & & & & & & & & & & & & \\
\hline $0 . C \%$ & $0.57^{* *}$ & $0.84^{* *}$ & 1 & & & & & & & & & & & & & \\
\hline Sand \% & -0.40 & -0.09 & -0.23 & 1 & & & & & & & & & & & & \\
\hline Clay $\%$ & 0.26 & -0.04 & 0.30 & $-0.44^{*}$ & 1 & & & & & & & & & & & \\
\hline Silt \% & 0.37 & 0.11 & 0.17 & $-0.97^{\text {** }}$ & 0.23 & 1 & & & & & & & & & & \\
\hline T.N\% & 0.58 * & $0.84^{* *}$ & $0.99^{* *}$ & -0.23 & 0.31 & 0.17 & 1 & & & & & & & & & \\
\hline $\begin{array}{l}\text { Total -P mg } \\
\mathrm{kg}^{-1}\end{array}$ & $0.62^{* *}$ & $0.47^{*}$ & $0.45^{*}$ & $-0.51^{*}$ & -0.01 & $0.55^{\text {*t }}$ & $0.44^{*}$ & 1 & & & & & & & & \\
\hline $\mathrm{Ca}-\mathrm{Pmg} \mathrm{kg}{ }^{-1}$ & 0.28 & -0.01 & 0.22 & $-0.46^{*}$ & 0.26 & $0.43^{*}$ & 0.22 & 0.59 * & 1 & & & & & & & \\
\hline $\mathrm{Fe}-\mathrm{P} \mathrm{mg} \mathrm{kg}^{-1}$ & $0.79^{\text {** }}$ & $0.63^{\text {*t }}$ & $0.64^{\text {*t }}$ & -0.33 & 0.15 & 0.32 & $0.64^{\text {* }}$ & $0.81^{\text {* }}$ & $0.42^{*}$ & 1 & & & & & & \\
\hline Al-P mg kg ${ }^{-1}$ & $-0.50^{\text {** }}$ & -0.29 & -0.25 & 0.08 & -0.02 & -0.08 & -0.24 & $-0.47^{\star}$ & -0.38 & $-0.66^{\text {** }}$ & 1 & & & & & \\
\hline $\begin{array}{l}\text { Available }-P \\
\mathrm{mg} \mathrm{kg}^{-1}\end{array}$ & 0.09 & 0.10 & -0.19 & 0.04 & -0.39 & 0.06 & -0.19 & 0.18 & -0.22 & -0.09 & 0.18 & 1 & & & & \\
\hline $\mathrm{Na} \mathrm{cmol} \mathrm{kg}^{-1}$ & -0.01 & -0.20 & -0.16 & -0.20 & 0.00 & 0.21 & -0.17 & 0.26 & 0.33 & -0.03 & 0.22 & -0.04 & 1 & & & \\
\hline Ca cmol kg ${ }^{-1}$ & $0.47^{*}$ & $0.72^{\text {*t }}$ & $0.75^{\text {*t }}$ & $-0.42^{*}$ & 0.12 & $0.42^{*}$ & 0.74 * & $0.60^{* *}$ & 0.24 & $0.66^{\text {** }}$ & -0.06 & 0.14 & -0.16 & 1 & & \\
\hline $\mathrm{Mg} \mathrm{cmol} \mathrm{kg}^{-1}$ & 0.48 * & $0.44^{*}$ & $0.49^{*}$ & $-0.63^{\text {** }}$ & 0.15 & $0.65^{\text {*t }}$ & $0.49^{*}$ & $0.67^{\text {** }}$ & 0.37 & $0.57^{* *}$ & 0.07 & 0.23 & 0.16 & $0.87^{* *}$ & 1 & \\
\hline $\mathrm{K} \mathrm{cmol} \mathrm{kg}^{-1}$ & $0.42^{*}$ & 0.26 & 0.35 & -0.02 & -0.10 & 0.05 & 0.35 & 0.29 & $0.44^{*}$ & 0.19 & -0.26 & 0.10 & 0.33 & 0.02 & 0.04 & 1 \\
\hline
\end{tabular}


Total nitrogen had a significant positive correlation with total phosphorus $(r=0.44)$, and with Iron bound phosphate $(r=0.64)$. There was also a positive correlation between total nitrogen percentage and calcium $(r=0.74)$, and with magnesium $(r=0.49)$. From the result, there was a significant positive correlation between calcium bound phosphate ( $\mathrm{Ca}-\mathrm{P})$ and iron bound phosphate (Fe-P) $(r=0.42)$, and also, exchangeable base potassium $(r=0.44)$. Iron bound phosphate (Fe-P) had a positive correlation with calcium ( $r=0.66)$, and magnesium $(r=0.57)$. However, iron bound phosphate had a negative significant correlation with aluminum bound phosphate $(\mathrm{Al}-\mathrm{P})(\mathrm{r}=-0.66)$.

\section{DISCUSSION}

The soil texture of the study areas within the depths of $0-30 \mathrm{~cm}$ and $30-60 \mathrm{~cm}$ can be classified as sandy soil (light-textured) as it constitutes majorly of sand particles compared to silt and clay. The results of the soil physical properties are in accordance with Oguntala (1980); Oyelowo et al (1998) and Isikhuemen (2005); Oladoye (2015). Similar trends in particle size distribution have been reported by Muoghalu and Awokunle (1994); Chima (2007). The electrical conductivity decreases with depths as $0-30 \mathrm{~cm}$ had the highest $(0.19 \pm 0.01 \mathrm{a}) 0.09 \pm 0.01 \mathrm{~b}$ in $30-60 \mathrm{~cm}$.

The soil $\mathrm{pH}$ values obtained in this study for both depths are similar to soil $\mathrm{pH}$ obtained by (Salim et al 2018). The soil pH is slightly acidic and near neutral. All the soils were acidic which agreed with Igwe (2001) who said that tropical soils tend to be acidic. Organic carbon also decreases down the depths. $0-30 \mathrm{~cm}$ had the highest organic carbon $17.00 \pm 0.13 \mathrm{ag} \mathrm{kg}{ }^{-1}$ and $30-60 \mathrm{~cm} 7.80 \pm 0.14 \mathrm{bg} \mathrm{kg}^{-1}$, which was as a result of the deposition of leaves and trees branches as a result of pruning on the upper layer of soil. The $\mathrm{pH}$ recorded in all the land use were slightly acidic and near neutral, but secondary forest soil showed a more moderately acidic soil, as the $\mathrm{pH}$ value recorded was slightly lesser than the rest. Significant difference observed in $\mathrm{pH}$ may be due to leaching of basic elements. Onyekwelu et al (2006) observed that nutrients are swiftly leached by heavy precipitation in the tropical rainforest. Decomposition of soil organic matter releases organic acids leading to decrease $\mathrm{pH}$ in forest (Killham 1994). This may be one of the reasons for having low soil pH under secondary forest as it produces large components of litter as compared to the other land uses. Similar observation was made by Hann (1997) who mentioned that the reduction in $\mathrm{pH}$ can be attributed to accumulation and subsequent slow decomposition of organic matter, which releases acids in the forest soil. In acidic soils, crystalline and non-crystalline oxides of Fe and Al (sesquioxides) are the main adsorbing agents of phosphate (Parfitt 1979).

The result of total nitrogen, calcium, sodium, magnesium, and potassium agreed with the result of (Oyelowo et al 2019). Total nitrogen decreases with depth. Calcium (Ca) is the dominating plant nutrient among the cations. It contributes to give the plant strong cellular walls, aid in the cell-division and is responsible for the activation of different enzymes (Marschner and Marschner 2012). According to Isikhuemen (2005), the presence of carbonates in soil can affect soil productivity by influencing soil $\mathrm{pH}$. The tree leaves and other plants components might have contributed significantly to the topsoil's levels of nitrogen, organic carbon, exchangeable calcium and magnesium. 
The total phosphorus in the depths of the studied soils were $99.51 \pm 3.21$ and $94.85 \pm 4.15$. The total phosphorus contents of normal soils vary from 100 to $2000 \mathrm{mg} \mathrm{kg}^{-1}$ (Kabata-Pendias and Pendias 1992). Hence, studied soils can be classified according to Tripathi et al (1970) as low in P $\left(<600 \mathrm{mg} \mathrm{kg}^{-1}\right)$. Total P is an inherent property of soils, and decreases with depth, similar to the findings of CadeMenun et al (2010) and Messiga et al (2012). Soil P occurs in inorganic and organic forms and their relative distribution varies with climate, vegetation, and parent material and soil management practices. For both depths, most of inorganic $P$ was present as Fe-P, followed by $\mathrm{Ca}-\mathrm{P}$, and then AI-P. Similar results were found in (Uriyo and Kasseba 1973, Udo and Ogunwale 1977, Abdel et al 2018). At both depths Fe-P fraction is high compared to Ca-P and Al-P in the soils; this may be due to the variation in climate, vegetation, and parent material and soil management practices (Pellerin et al 2006, Karazawa and Tabeke 2012).

Higher total nitrogen content in Tectona grandis, above Leucena leucocephana in this study is contrary to the finding of Awopegba et al (2017) that it is an established fact that Leucaena leucocephala being a legume is a good nitrogen fixer. The high total nitrogen observed in Tectona grandis could be as a result of the high deposition of litter on the surface soil of the plantation.

According to the rating by Tekalign (1991), the soil OC contents were in moderate to high range. However, SOC contents of all the soils were in the high range 0.17 to $0.25 \%$. Tectona grandis plantation recorded the highest organic carbon content; this could be due to the high litter deposit followed by Leucaena leucocephala plantation and the least in secondary forest. The exchangeable concentrations of $\mathrm{Ca}, \mathrm{Mg}$, and $\mathrm{K}$ in the various land use are noteworthy. Baillie and Aghton (1983) suggested that $\mathrm{Ca}$ and $\mathrm{Mg}$ should be more variable than $\mathrm{K}$ in forest soils. In the four-land use, exchangeable Calcium ( $\mathrm{Ca}$ ) tends to be more abundant, followed by magnesium (Mg). Leucaena leucocephala plantation recorded the highest exchangeable calcium and magnesium; similar results have been reported by Fasina et al $(2006,2015)$. On the other hand, an increase in soil pH along the soil depth indicates accumulation of bases (Kumar et al 2012). Total phosphorus was observed to be higher in Leucaena plantation soils and can be classified according to Tripathi et al (1970) as low in $P\left(<600 \mathrm{mg} \mathrm{kg}^{-1}\right)$. Because total $P$ is an inherent property of soils, the generally low total $P$ values in studied soils could be related to the nature of the parent material. Soil P occurs in inorganic and organic forms and their relative distribution varies with climate, vegetation, parent material, and soil management practices. Total phosphorus content of soils is of little importance for soil fertility, but it has been used as weathering index to differentiate between highly weathered and less weathered soils (Walker and Syers 1976). It is also used as a check on $\mathrm{P}$ balance when soil $\mathrm{P}$ is fractionated.

Soil $P$ exists in various chemical forms including inorganic Phosphorus and organic Phosphorus. These P forms differ in their behavior and fate in soils, and Phosphorus fractions usually account for $35 \%$ to $70 \%$ of total Phosphorus in soil (Turner et al 2003, Hansen et al 2001). With increasing soil pH, solubility of Fe and Al phosphates increase, but solubility of $\mathrm{Ca}$ phosphate decreases, except for $\mathrm{pH}$ values above 8 (Hinsinger 2001). In this study, the Ca-P fraction in all the study area is similar to the findings of Mostashari et al (2008). Generally, Fe-P tends to be the highest in the entire plantations in relation to Al-P and Ca-P in the study area. The high concentration of Fe-P in the study areas could be due to the soil parent 
materials and soil management practices. The very high contents of Fe-P among the active inorganic $\mathrm{P}$ fractions compared to the other fractions is due to the high content of Fe-oxides, acidic $\mathrm{pH}$, and advanced stage of weathering (Asmare et al 2015).

Available phosphorus was high in Leucaena plantation compared to others. The high available phosphorus may be due to the high proportion of exchangeable bases, electrical conductivity, organic carbon, and the relative soil $\mathrm{pH}$ in the soil of Leucaena plantation.

The particle size distribution shows that sand particles content in all the land use were high, and it was the same in the depths of Gmelina, Leucaena plantation and secondary forest. However, sand particles decreased with depth in Tectona grandis plantation. Sand particles were high in both depths $(0-30 \mathrm{~cm}$ and $30-60 \mathrm{~cm})$ of secondary forest and in the $0-30 \mathrm{~cm}$ depth of Tectona plantation; high sand particles in the $0-30 \mathrm{~cm}$ of Tectona grandis can be related to the large amount of organic matter deposited on the soil surface. Clay particles varied with depth in Gmelina and Tectona plantation, while it was constant in depths of Leucaena plantation and secondary forest. Clay particles $(8 \pm 0.58 \mathrm{a})$ were high in Gmelina plantation $(0-30 \mathrm{~cm})$ and Tectona grandis plantation $(30-60 \mathrm{~cm})$. Silt particles increased with depth in Gmelina and Tectona plantation and were constant in both depths of Leucaena and secondary forest. Similar trends in particle size distribution have been reported by Muoghalu and Awokunle (1994). The results of the soil physical properties agree with Oguntala (1980); Isikhuemen (2005) and Faboya et al (2015).

The $\mathrm{pH}$ values decreased with depth in Gmelina arborea, Tectona grandis plantation and secondary forest, while it increased with depth in Leucaena plantation. The soil $\mathrm{pH}$ in Gmelina and Tectona plantation both at $0-30 \mathrm{~cm}$ was neutral. Others varied from slightly acidic to moderately acidic. Higher $\mathrm{pH}$ values recorded in nearly all the depths might be due to the presence of higher exchangeable $\mathrm{Ca}$ and $\mathrm{Mg}$ in the soil. According to Isikhuemen (2005) the presence of carbonates in soil can affect soil productivity by influencing soil $\mathrm{pH}$. This result is in accordance with the findings done by (Faboya et al 2015).

Organic carbon percentage decreased with depth in the soil of Gmelina and Leucaena plantation and secondary forest, while it remained constant along the depth in the soil of Tectona plantation. The constant distribution of organic carbon in the soil of Tectona plantation is due to the equal deposition of organic matter in the soil. According to the rating by Tekalign (1991), the soil organic carbon contents were in moderate to high range in the various depths $(0-30$ and $30-60 \mathrm{~cm})$. The relatively high organic carbon content in the soil of all the land-use may be due to decayed products of underground root biomass which predictably was higher. The soil organic matter accumulation has been shown to be proportional to the extent of canopy closure (Gupta et al 1991). The occurrence of higher topsoil organic carbon in all the study areas can be due to the litter fall addition from trees and shrubs to the surface soil (Nsabimana et al 2008). The mean value of organic matter content in the study areas was high in Leucaena planation $0-30 \mathrm{~cm}(2.24 \pm 0.06 \mathrm{a})$ and in Gmelina plantation $0-30 \mathrm{~cm}(1.86 \pm 0.06 \mathrm{~b})$. In all the land-use, organic carbon and total nitrogen were abundant in the $0-30 \mathrm{~cm}$ depth, which is a result of tree leaves and other plant components contributing significantly to the topsoil's levels of nitrogen, organic carbon, exchangeable calcium, and magnesium (Oyelowo et al 2019). 
The exchangeable bases varied between the depth in each study areas. Calcium (Ca) decreased from $0-30 \mathrm{~cm}$ to $30-60 \mathrm{~cm}$ depth; magnesium $(\mathrm{Mg}$ ) content decreased with depth from $0-30 \mathrm{~cm}$ to $30-60 \mathrm{~cm}$ in Gmelina plantation and Leucaena plantation, while it increased with depth down the depths in Tectona plantation and secondary forest. The exchangeable concentration of calcium, magnesium, sodium and potassium in the various depth intervals is noteworthy. Baillie and Aghton (1983) suggested that calcium and magnesium should be more variable than potassium in forest soils, as potassium occurs exclusively on soil organic matter exchange sites.

Total phosphorus content of soils is of little importance for soil fertility, but it has been used as weathering index to differentiate between highly weathered and less weathered soils (Walker et al 1976). It is also used as a check on P balance when soil $P$ is fractionated. Generally, the amount of inorganic total phosphorus decreased with increase in depth of soil, but this was the case for all the study area soils where the soil $(0-30 \mathrm{~cm})$ recorded higher amount than soil of $(30-60 \mathrm{~cm})$. Leucaena plantation had the highest total phosphorus $(111.45 \pm 3.32 \mathrm{a})$ at depth of $0-30 \mathrm{~cm}$ and the lowest in the secondary forest $(79.85 \pm 10.77 \mathrm{c})$ at the depth of $30-60 \mathrm{~cm}$, which could be due to erosion, crop removal, and precipitation of phosphorus from soil solution which decreases Phosphorus availability. Ali et al (2010) also obtained a phosphorus content that decreased down the profile. A study by Acquaye and Beringer (1989) in the savannah zone of Ghana showed that Ca-P constituted $75 \%$ of the inorganic $P$ fractions and $\mathrm{Ca}$ being the predominant cation on the exchange complex of that soil means most of the fertilizer $\mathrm{P}$ was transformed into $\mathrm{Ca}-\mathrm{P}$. The Ca-P fraction is low compared to the findings from this study, as Fe-P was found to be dominant in all the depth of Gmelina, Tectona, Leucaena plantation and secondary forest in which the highest Fe-P was found in Gmelina plantation $(48.1 \pm 2.31 \mathrm{a})$ at $0-30 \mathrm{~cm}$ and $47.08 \pm 2.68 \mathrm{a}$ in Leucaena plantation at $0-30 \mathrm{~cm}$ depth. Fe-P and Al-P fraction of phosphorus were closely similar both having $28.19 \pm 5.71 \mathrm{c}$ and $28.05 \pm 7.54$ a respectively at $30-60 \mathrm{~cm}$ depth in secondary forest, while it varied in others.

Available phosphorus observed in each study area was relatively higher compared to the result of Faboya et al (2015) in mixed plantations.

Correlation studies above showed that $\mathrm{pH}$ and the other physicochemical parameters influence the formation of Fe-P fractions and total phosphorus. Also, the correlation between calcium bound phosphate (Ca-P) and iron bound phosphate (Fe-P) might be due to the increase in chemical weathering of $P$ containing parent materials which will precipitate calcium and iron. In addition, the physicochemical parameters have significant effects on the Total-P. Correlation between total phosphorus, iron bound phosphate and calcium bound phosphate shows that the parent material present contains high concentration of calcium and iron. The effect of silt on Ca-P and the exchangeable base calcium might be due to chemical weathering of Phosphorus bearing calcium mineral.

The study also shows that sand does not influence the formation of phosphorus fractions and has little effect on other physiochemical properties. This might be responsible for the low total phosphorus and available phosphorus because a high percentage of sand particles was recorded in the depths of all the land use, and sand is known to have low adsorption of phosphorus fractions due to the low surface area. 


\section{CONCLUSION}

The study revealed that the level of inorganic Phosphorus in soils decreased with increase in depth while Fe-P was dominant at all the depths. The trend for phosphorus fraction at both depth $(0-30 \mathrm{~cm}$ and $30-60 \mathrm{~cm})$ in secondary forest is as follows (Fe-P>Al-P>Ca-P): Gmelina arborea plantation Phosphorus fractions (Fe$\mathrm{P}>\mathrm{Ca}-\mathrm{P}>\mathrm{Al}-\mathrm{P}$ ), Tectona grandis plantation ( $\mathrm{Fe}-\mathrm{P}>\mathrm{Ca}-\mathrm{P}>\mathrm{Al}-\mathrm{P}$ ), and Leucaena leucocephala plantation (Fe-P>Ca-P>Al-P). In the soils studied, $\mathrm{Ca}-\mathrm{P}$ and $\mathrm{Al}-\mathrm{P}$ were relatively low, but Fe-P was generally high. Leucaena plantation had the highest total phosphorus, followed by Gmelina plantation and by Teak plantation; the least was in the secondary forest. Lower nutrient concentrations and contents in soils under secondary forest within the depths were due to lower OM content, and probably due to higher nutrient demand and nutrient immobilization by tree species and other flora present. P fractionation investigations are therefore recommended in soils under indigenous species plantation in order to provide adequate understanding of $\mathrm{P}$ status in the soils and fertilizer recommendation so as to improve productivity. Finding of this study is also recommended for proper soil management practices in the study area to improve availability of phosphorus and other nutrients.

\section{REFERENCES}

Abdel WA, Mohamed A, Mohammed AE, Yousif E \& Gorashi EM. 2018. Relationship between phosphorus fractions of some selected Sudanese soil orders to phosphate availability. Eurasian Journal of Soil Science 7(3):224-229

Acquaye DK and Beringer H. 1989. Sulfur in Ghanaian soils. Plant and Soil 113:197-203

Ali A, Ali Z, Iqbal J, Nadeem MA, Akhtar N, Akram HM \& Sattar A. 2010. Impact of nitrogen and phosphorus on seed yield of chickpea. Journal of Agricultural Research 48(3):335-342

Asmare M, Heluf G, Markku Y \& Birru Y. 2015. Phosphorus Status, Inorganic Phosphorus Forms, and Other Physicochemical Properties of Acid Soils of Farta District, Northwestern Highlands of Ethiopia. Applied and Environmental Soil Science 2015:1-11

Awopegba M, Oladele S \& Awodun M. 2017. Effect of mulch types on nutrient composition, maize (Zea mays L.) yield and soil properties of a tropical Alfisol in Southwestern Nigeria. Eurasian Journal of Soil Science 6(2):121-133

Baillie IC and Ashton PS. 1983. Some soil aspects of the nutrient cycle in mixed dipterocarp forests in Sarawak. In Sutton SL, Whitmore TC \& Chadwick AC (eds) Tropical rain forest: ecology and management (pp347-356).Blackwell Press: Oxford, UK

Bouyoucos GJ. 1962. Hydrometer Method Improved for Making Particle Size Analysis of Soils. Agronomy Journal 54(5):464-465

Busman L, Lamb J, Randall GR \& Schmit M. 2002. The nature of phosphorus in soils. University of Minnesota Extension Service

Cade-Menun BJ, Carter MR, James DC \& Liu CW. 2010. Phosphorus forms and chemistry in the soil profile under longterm conservation tillage: a phosphorus 31 nuclear magnetic resonance study. Journal of Environmental Quality 39(5):1647-1656 
Chang SC and Jackson ML. 1957. Fractionation of soil phosphorus. Soil Science 84(2):113-144

Chang SC and Jackson ML. 1958. Soil phosphorus fractionation in some representative soils. European Journal of Soil Science 9(1):109-119

Chima UD. 2007. Effects of tropical rainforest modification on soil quality of Omo biosphere reserve in Ogun State, Nigeria (MS thesis). University of Agriculture, Abeokuta, Ogun state, Nigeria

Dean HT. 1938. Endemic fluorosis and its relation to dental caries. Public Health Report 53(33):1443-1452

Duncan DB. 1955. Multiple range and multiple f-test. Biometrics 11(1):1-5

Faboya OL, Oso AO, Aladesanmi AO \& Osasona I. 2015. Heavy metal concentrations in soils adjoining some refined petroleum products facilities in Ado-Ekiti, Nigeria

Fasina AS, Shittu OS, Omotoso SO \& Adenikinju AP. 2006. Response of cocoa to different fertilizer regimes on some selected soils of Southwestern Nigeria. Agriculture Journal 1(4):272-276

Fasina AS, Raji A, Oluwatosin GA, Omoju OJ \& Oluwadare DA. 2015. Properties, genesis, classification, capability and sustainable management of soils from South-Western Nigeria. International Journal of Soil Science 10(3):142-152

Gupta SC, Lowery B, Moncrief JF \& Larson WE. 1991. Modeling tillage effects on soil physical properties. Soil and Tillage Research 20(2-4):293-318

Hansen B, Fjelsted Alrøe H \& Kristensen E. 2001. Approaches to assess the environmental impact of organic farming with particular regard to Denmark. Agriculture, Ecosystems \& Environment 83(1-2):11-26

Havlin JL, Betson JD, Tisdale SL \& Nelson WL. 1995. Soil fertility and fertilizers (6th edn). Prentice-Hall, Upper Saddle River, New York, USA

Hinsinger P. 2001. Bioavailability of soil inorganic $P$ in the rhizosphere as affected by root-induced chemical changes: a review. Plant and Soil 237(2):173-195

Igwe CA. 2001. Free oxide distribution in Niger flood plain soils in relation to their total and available phosphorus. Proceedings of the 27th Annual Conference of the Soil Science Society of Nigeria, IEEE Xplore, London

Isikhuemen EM. 2005. Tree population dynamics and regeneration of a logged-over secondary regrowth forest (PhD dissertation). University of Agriculture, Abeokuta, Nigeria

Jackson ML. 1958. Soil chemical analysis. Prentice Hall, New York

Junbao Y, Kai N, Yunzhao L, Siyao D, Guangxuan H, Qinghui X, Huifeng W, Guangmei W \& Yongjun G. 2014. Wet and dry atmospheric depositions of inorganic nitrogen during plant growing season in the coastal zone of yellow river delta. Scientific World Journal 2014:1-8

Kabata-Pendias A and Pendias H. 1992. Trace elements in soils and plants (2nd edn). CRC Press, Boca Raton

Karazawa T and Tabeke M. 2012. Temporal or spatial arrangements of cover crops to promote arbuscular mycorrhizal colonization and $\mathrm{P}$ uptake of upland crops grown after nonmycorrhizal crops. Plant and Soil 353(1-2):355-366

Killham K.1994. Soil ecology. Cambridge: Cambridge University Press

Kumar YS and Naidu MVS. 2012. Characteristics and classification of soils representing major land forms in Vadamalalpeta Mandal of Chittoor District, Andhra Pradesh. Journal of the Indian Society of Soil Science 60(1):63-67 
Marschner H and Marschner P. 2012. Marschner's mineral nutrition of higher plants (3rd edn). London. Waltham, MA: Elsevier/Academic Press

Messiga AJ, Ziadi N, Be'langer G \& Morel C. 2012. Process-based mass-balance modeling of soil phosphorus availability in a grassland fertilized with $\mathrm{N}$ and $\mathrm{P}$. Nutrient Cycling Agroecosystems 92(3):273-287

Mostashari M, Muazardalan M, Karimian N, Hosseini HM \& Rezai H. 2008. Phosphorus fractions of selected calcareous soils of Qazvin province and their relationships with soil characteristics. American-Eurasian Journal Agricultural and Environmental Sciences 3(4):547-553

Muoghalu JI and Awokunle HO. 1994. Spatial Patterns of Soil Properties under Tree Canopy in Nigeria Rain Forest Region. Tropical Ecology 35(2):219-228

Murphy $J$ and Riley JP. 1962. A modified single solution method for the determination of phosphate in natural waters. Analytica Chimica Acta 27:31-36

Nelson DW and Sommers LE. 1996. Total carbon, organic carbon, and organic matter. In Sparks DL, Page AL, Helmke PA, Loeppert RH, Soltanpour MA, Tabatbai MA, Johnston CT \& Summer ME (eds) Methods of Soil Analysis: Part 3 (pp961-1010). SSSA Book Series, Madison

Nsabimana D, Klemendtson L, Kaplin BA \& Wallin G. 2008. Soil carbon and nutrient accumulation under forest plantations in southern Rwandan. African Journal of Environmental Science and Technology 2:142-149

Oguntala AB. 1980. The effects of fire on aspects of nitrogen cycling in Olokemeji Forest Reserve, Nigeria. Royal Swedish Academy of Sciences for SCOPE/UNEP International Nitrogen Unit

Oladoye AO. 2015. Physicochemical properties of soil under two different depths in a tropical forest of international institute of tropical agriculture, Abeokuta, Ibadan, Nigeria. Journal of Research in Forestry, Wildlife and Environment 7(1):40-54

Onyekwelu J, Mosandl R \& Stimm B. 2006. Productivity, site evaluation and state of nutrition of gmelina arborea plantations in Oluwa and Omo forest reserves, Nigeria. Forest Ecology and Management 229(1-3):214-227

Oyelowo O, Aduradola A, Sulaiman O \& Aina-oduntan OA. 2019. Physico-chemical characteristics of soils in two sacred groves of Western Nigeria. Ethiopian Journal of Environmental Studies \& Management 12(4):462-471

Pellerin A, Parent LE, Fortin J, Tremblay C, Khiari L \& Giroux M. 2006. Environmental mehlich-III soil phosphorus saturation indices for quebec acid to near neutral mineral soils varying in texture and genesis. Canadian Journal of Soil Science 86(4):711-723

Salim MVC, Miller RP, Ticona-Benavente CA, van Leeuwen J \& Alfaia SS. 2018. Soil fertility management in indigenous homegardens of Central Amazonia, Brazil. Agroforestry Systems 92(2):463-472

Sam-Wobo S and Mafiana C. 2006. The effects of surface soil physico-chemical properties on the prevalence of helmiths in Ogun State, Nigeria. Journal of Science and Technology (Zambia) 9(2):13-20

SAS. 2003. Statistical Analysis System. SAS Release 9.1 for windows, SAS Institute Inc.Cary, NC, USA

Tekalign T. 1991. Soil, plant, water, fertilizer, animal manure and compost analysis. Working Document No. 13, International Livestock Research Center for Africa, Addis Ababa, Ethiopia, 1991 
Thomas GW. 1996. Soil pH and soil acidity. In Sparks DL, Page AL, Helmke PA, Loeppert RH, Soltanpour PN, Tabatabai MA, Johnson CT, Summer ME (eds) Chemical Methods of Soil Analysis Part 3 (pp475-490). Soil Science Society of American Book series, American Society of Agronomy, Madison USA

Tiyapongpattana W, Pongsakul P, Shiowatana J \& Nacapricha D. 2004. Sequential extraction of phosphorus in soil and sediment using a continuous-flow system. Talanta 62(4):765-771

Tripathi BR, Tandon HLS \& Tyner EH. 1970. Native inorganic phosphorus forms and their relation to some chemical indicies of phosphate availability for soils of Agra district, India. Soil Science 109(2):93-101

Turner BL, Mahieu N \& Condron LM. 2003. Phosphorus-31 nuclear magneti resonance spectral assignments of phosphorus compounds in soil $\mathrm{NaOH}-E D T A$ extracts. Soil Science Society American Journal 67(2):497-510

Udo EJ and Ogunwale JA. 1977. Phosphorus fractions in selected Nigerian soils. Soil Science Society American Journal 41(6):1141-1146

Uriyo AP and Kasseba A. 1973. Phosphate fraction in some Tanzania soils. Geoderma 10:181-192

Walker TW and Syers JK. 1976. The fate of phosphorus during pedogenesis. Geoderma 15(1):1-19

Walkley A and Black IA. 1934. An examination of the degtgareff method of determination of organic matter and proposed modification of chromic acid titration method. Soil Science 37:29-38 